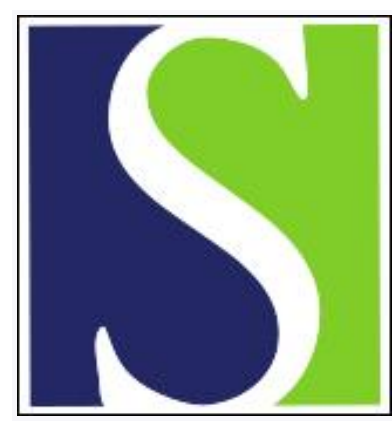

Scand J Work Environ Health 1990;16(1):59-66

https://doi.org/10.5271/sjweh.1820

Issue date: 1990

Perception of effort in manual materials handling.

by Gamberale F

Affiliation: National Institute of Occupational Health, Solna, Sweden.

The following article refers to this text: 2012;38(6):582-589

This article in PubMed: www.ncbi.nlm.nih.gov/pubmed/2189222 


\title{
Perception of effort in manual materials handling
}

\author{
by Francesco Gamberale, PhD ${ }^{1}$
}

\begin{abstract}
GAMBERALE F. Perception of effort in manual materials handling. Scand $J$ Work Environ Health 1990;16(suppl 1):59-66. Measurement of subjective work load has emerged as a central topic of interest in the study of physical work and effort. From the practical point of view, subjective estimates of physical effort can contribute greatly to the assessment of work loads in physical activities and to the design of manual handling tasks. The rationale of this approach is that subjective estimates reflect the individual perception of the amount of physical and motivational resources that the subjects invest to meet the demand imposed by the physical task. The present paper provides an illustration of some of the techniques used in measuring the perception of physical effort and reviews and discusses the main results obtained with these techniques in the assessment of manual materials handling.
\end{abstract}

Key terms: maximum acceptable work load, perceived exertion, physical work.

From the physiological point of view, individual capacity to perform physical work is limited by central factors associated with the pulmonary, circulatory, and metabolic systems and by local factors concerning muscular strength, joints, and the skeleton. On the basis of these considerations many biomechanical and physiological methods have been developed to predict the work capacity of individuals or to assess the physical demands of specific manual handling tasks.

From the psychological point of view, motivational factors play an important role in the perception of effort and, consequently, in determining the individual capacity for physical work.

Subjective reactions to physical work, such as perceived effort or perceived work load, have often been found to correlate with work intensity and work performance. Until recently, however, subjective measures of fatigue have not been seriously considered as constituting a possible basis for criteria in the assessment of manual materials handling. The major reason for the neglect of subjective reactions in favor of physiological indicators of fatigue is that these reactions have been difficult to define and measure. Another reason has been the lack of familiarity with the use of "sophisticated" psychophysical methods on the part of the physiologist. However, some of the difficulties encountered in the use of subjective indicators of fatigue are fundamental and are connected with the definition of the subjective dimensions involved and with the nature of the measurement itself. Thus subjective reaction to physical work can only be measured indirectly through the use of self-reported techniques, and sometimes it is not possible to ascertain to what extent the response obtained reflects perceptual or cognitive processes.

I National Institute of Occupational Health, Solna, Sweden.

Reprint requests to: Professor $\mathrm{F}$ Gamberale, National Institute of Occupational Health, S-171 84 Solna, Sweden.
The suitability of subjective indicators of fatigue as criteria in the assessment of manual materials handling will always depend on a series of elements which affect the reliability and validity of the measurements, eg, (i) the type of subjective reaction observed, (ii) the way in which the reaction is observed and recorded, (iii) the extent to which the reaction varies systematically in different work operations, (iv) how well the reaction correlates with work intensity and work performance, (v) how well the reaction correlates with the physiological and neurological events, and (vi) the extent to which the reaction can be predicted from knowledge of the individual and of the work operations.

An analysis of the literature clearly shows that in recent years the study of the subjective reactions to physical work has relied almost exclusively on a few types of methods, eg, (i) magnitude estimation, (ii) ratings of perceived exertion, (iii) ratings of effort expended, and (iv) ratings of acceptable load. The psychophysical methods, mainly magnitude estimation, have been mostly used in basic research with the aim of studying the relation between the physical or objective work load and the subjective work load. [See the paper by Borg (1)]. The other methods have, however, been more widely used in applied ergonomics, and these will be described in the present paper, in which the main results obtained through their application will also be reviewed.

\section{Magnitude estimation}

In magnitude estimation the subject is required to match a number directly to the perceived intensity of a physical exercise in reference to a standard intensity which has a given numerical magnitude. The basic assumption of this direct-scaling method is that the subject is able to match his perception with numbers. Numerous empirical studies have shown that subjects 
are able to perform this task and that the subjective scale obtained is a ratio scale.

The results of several psychophysical studies using magnitude estimation clearly indicate that the perceived intensity of muscular effort is a power function of the physical work load. [See also the paper by Borg (1)]. Thus Stevens \& Mack (2) obtained a power function with an exponent of 1.7 when scaling subjective force, as exerted in the squeezing of a handle. Borg \& Dahlström (3) investigated muscular work carried out on a bicycle ergometer and obtained an exponent of 1.6. Exponents of the same magnitude were also found by Eisler (4) in relating subjective force to physical force both for work involving large muscle groups, ie, exerting a force against a foot pedal, and for work involving small muscle groups, ie, squeezing a handgrip. A power function with an exponent of 1.6 was also obtained by my co-workers and I (5) in an investigation of the subjective experience associated with external resistance to breathing.

Perceived effort depends not only on the intensity of the physical work performed, but also on the duration of the work. When investigating the combined effects of the two factors, Stevens \& Cain (6) and, later, Caffarelli et al (7) found that the increase in effort with time also followed a power function. The exponent of this function was shown to depend on the type of work investigated and on its intensity.

Table 1. Magnitude estimates (ME) of perceived work load as a function of case weight in the horizontal lifting of cases [from reference $(8)]$.

\begin{tabular}{lll}
\hline Subject & Function & $\mathrm{R}^{2}$ \\
\hline 1 & $\mathrm{ME}=0.010 \mathrm{~kg}^{1.643}$ & 0.969 \\
2 & $\mathrm{ME}=0.032 \mathrm{~kg}^{1.444}$ & 0.991 \\
3 & $\mathrm{ME}=0.015 \mathrm{~kg}^{1.702}$ & 0.989 \\
4 & $\mathrm{ME}=0.006 \mathrm{Kg}^{2.061}$ & 0.979 \\
5 & $\mathrm{ME}=0.011 \mathrm{~kg}^{2.449}$ & 0.998 \\
\hline Total & $\mathrm{ME}=0.011 \mathrm{~kg}^{1.860}$ & 0.997 \\
\hline
\end{tabular}

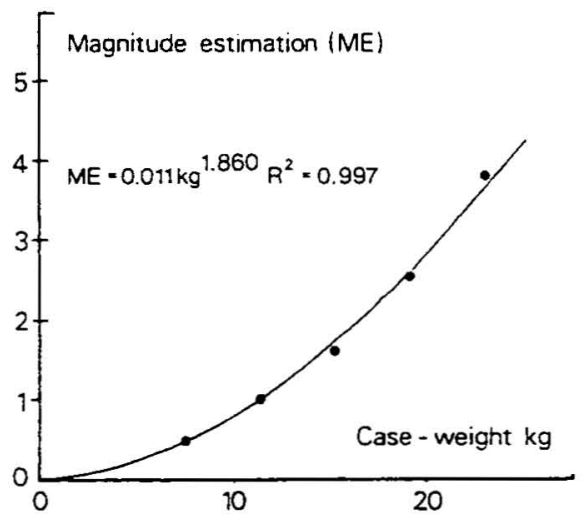

Figure 1. Magnitude estimation (ME) of perceived work load as a function of case weight. The plotting and curve fitting were performed on the basis of average values for the five subjects. [Adapted from Ljungberg et al (8)]
To give a more concrete example of the application of ratio scaling to the study of perceived exertion in manual materials handling, some of the results of an experiment performed in the laboratory (8) will be referred to in more detail.

In this experiment five brewer's draymen with several years of occupational experience were studied in the laboratory during horizontal lifting work, which simulated an order filling task at a brewery. The task consisted of lifting a case $(36 \times 36 \times 24 \mathrm{~cm})$ approximately $1.1 \mathrm{~m}$ horizontally at a height of $60 \mathrm{~cm}$. In order to facilitate repeated lifting, a treadmill was used to return the case after each lift. The work was performed in cycles of three consecutive lifts followed by a rest period. The subjects worked at a given lifting rate with cases weighing $67,100,133,167$, and $200 \%$ of a reference case weight, which they had individually chosen as constituting the maximum amount that they could lift without strain and discomfort. (See the section Acceptable Load.) After $11 \mathrm{~min}$ of work at each load, each subject was asked to give a magnitude estimation of the work load. The estimations were made by having the subject indicate a number equivalent to the perceived work load in relation to the immediately preceding work load, which was always assigned the value 100 . The sequence of work loads was chosen in such a way that when a subject had compared each load with the immediately preceding load, he had performed all possible pairwise comparisons between the loads. Since the sequence of the work loads was also performed in reverse order, a complete matrix of relative comparisons between case weights was obtained for each subject. On the basis of these matrices, a scale of perceived work load was calculated for each subject according to Ekman (9). With the same methods, a subjective scale was also calculated for all the subjects on the basis of a ratio matrix of geometric mean values. The results of this part of the investigation are illustrated in table 1 and figure 1 . As shown, the relation between the magnitude estimates of perceived work load and case weight (objective load) is satisfactorily described by the psychophysical power function. The exponents of the individual subject functions varied between 1.44 and 2.45 , indicating that a doubling of the case weight would result in an approximately three- to fivefold increase in perceived work load.

The same technique of collecting magnitude estimations and producing subjective scales of physical work load was also used by my co-workers and I (10) in an experiment in which a complex, diagonal lifting task was studied. (See the section Acceptable Load.) In this experiment the subjective work load was also a power function of the physical work load, as illustrated in figure 2 .

In summary, the application of the psychophysical method of magnitude estimation to the study of physical work can provide a detailed description of the relationship between the perceived and the physical level 

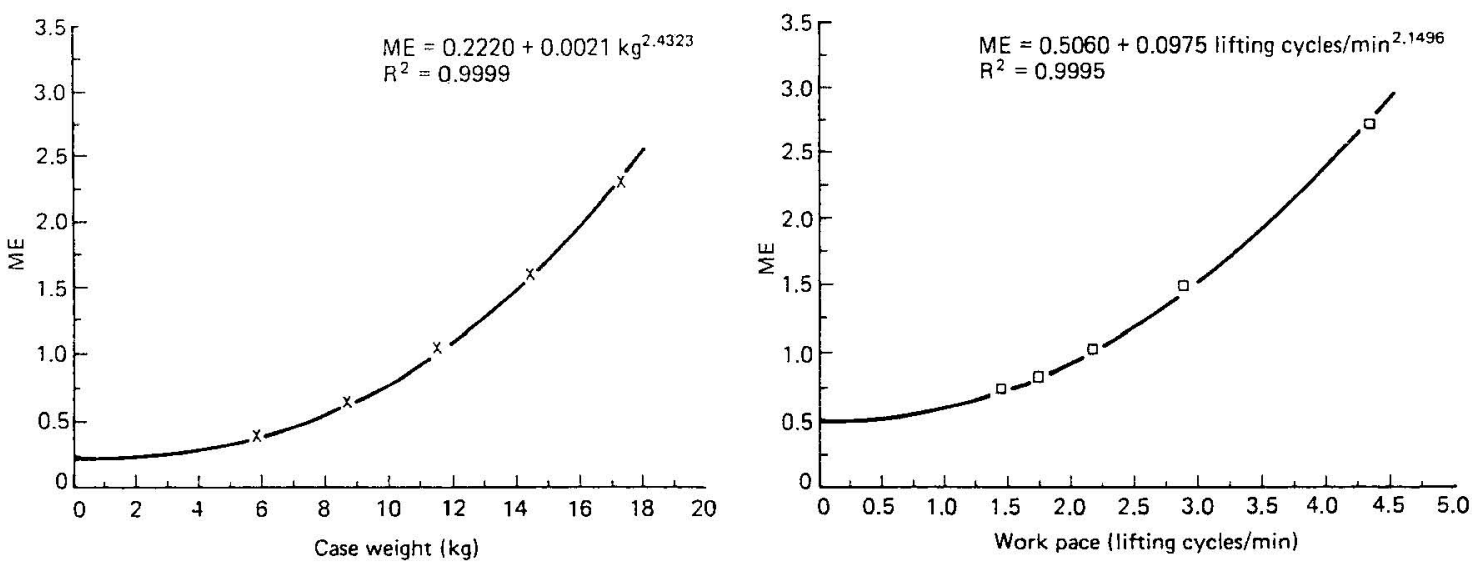

Figure 2. Magnitude estimation (ME) of perceived work load as a function of case weight and as a function of workpace. The plotting and functional fit were performed on the basis of average values for 16 subjects. [Adapted from Gamberale et al (10)]

of exertion. There is strong empirical evidence that perceived exertion is a positively accelerated function of work load, irrespective of the type of work performance or the muscle groups involved.

\section{Perceived exertion}

By far the most important contribution in the study of the psychological aspects of physical work has been made by Borg $(11,12)$, who is also the author of the most frequently used scale for rating the degree of perceived exertion during physical work, the so-called RPE scale (rating of perceived exertion scale) (13). The scale, printed in a quarto format, is as follows:

6

7 Very, very light

8

9 Very light

10

11 Fairly light

12

13 Somewhat hard

14

15 Hard

16

17

18

19 Very, very hard

20

The RPE scale has been developed on the basis of empirical data from work on the bicycle ergometer. "In fairly young to middle-aged people ( 25 to 45 years old), working at moderate to high intensity levels, the heart rate roughly corresponds to 10 times the RPE value [p 341]" (14). Thus, according to Borg, the relation between RPE and heart rate for work on the bicycle ergometer is linear. Consequently, RPE is also a linear function of the physical work load. This conclusion should not be interpreted as conflicting with the results of several psychophysical studies, in which perceived exertion was found to be a positively accelerated function of the work load. It is important to observe that the form of the relation obtained when RPE values are plotted against the corresponding values of heart rate for the same work load depends largely on the specific characteristic of the rating scale itself, ie, the number of categories, the verbal definition, etc. One of the objectives in the construction and development of the scale was in fact to obtain a linear relationship between the RPE and work load. This objective was achieved by a careful choice of verbal categories.

The RPE scale has been the most frequently employed method for the assessment of subjective qualities during physical work. Thus a substantial volume of literature has become available concerning the significance of perceived exertion as measured by the RPE scale. Since RPE values could be used as a complement to circulatory responses during exercise, and in fact the scale was constructed with this objective, it is of particular interest to observe circumstances when changes in physiological responses are not followed by corresponding changes in RPE.

In a study by Pandolf et al (15), changes in temperature were found to affect heart rate but not RPE. In another study (16) the heat gain resulting from wearing an unventilated gas-protective suit during simulated firefighting and gas accident practice, as well as during exercise on a bicycle ergometer, brought about a considerable increase in heart rate which was not followed by a corresponding increase in RPE. These results suggest that heat load does not have an impact upon perceived exertion that is comparable to the physiological strain. it produces. Thus heat load may 
create conditions in which a person will overestimate his or her physical endurance. An overestimation of this kind could be a determinant factor in the occurrence of cases of exhaustion and collapse observed in firefighting and other extreme situations (16). Provided that exercise on the bicycle ergometer is performed at the same work load, eg, $100 \mathrm{~W}$, different pedaling rates produce the same heart rate. However, Henriksson et al (17) and Pandolf \& Noble (18) have reported that pedaling at a rate of 30 or 40 revolutions $/ \mathrm{min}$ resulted in a higher RPE than pedaling at a rate of 60 or 80 revolutions/min. Ekblom \& Goldbarg (19) and Sjöberg \& Frankenhaeuser (20) used autonomic blocking drugs to affect heart rate during physical work. While heart rate changed in the expected direction as a result of the drugs, RPE was unaffected. Pandolf et al (15) and Morgan (21) reported that while heart rate was unchanged during the course of work on a bicycle ergometer, RPE tended to increase after 5 min of work. Similar results were obtained by Ljungberg et al (8) during horizontal lifting work. RPE collected after both 4 and $13 \mathrm{~min}$ of work showed a significant increase, while heart rate did not display any noticeable differences. Finally, my co-workers and I (22) showed that, at the same heart rate, exercise on a bicycle ergometer was perceived by a group of women as more demandirg during menstruation than during either the premenstrual or postmenstrual phase. The changes in RPE during the menstrual cycle were interpreted as due to motivational factors.

Many investigations have shown that the relationship between RPE and heart rate is highly dependent upon the type of physical task involved. At a given heart rate, RPE is lower for running than for work on the bicycle ergometer $(19,23)$ or for walking $(24)$. At the same heart rate or at the same oxygen uptake, arm exercise also gives a higher RPE than cycling or pushing a wheelbarrow $(19,25)$. Changes in RPE were also associated with increases in blood lactate concentration, which led to the formulation of the following hypothesis: "The higher the blood lactate concentration an exercise produces as compared to oxygen uptake, the higher will be the level of the overall perception of exertion... [p 553]" (25). In addition to the RPE scale, other rating scales have been used for measuring perceived exertion. However, not one of these scales has shown the same versatility, parsimony, and validity as the RPE scale.

\section{Expended effort}

In several investigations subjective fatigue during prolonged physical exercise and endurance tasks has been studied while the subjects rate the degree of effort expended over time. The technique used was developed by Caldwell (26) for use with an isometric handgrip task. The procedure consists of letting the subjects indicate when, in an endurance task, they per- ceive the expenditure of one-fifth, two-fifths, etc, of their available effort or when, in the same self-paced fashion, pain intensity attains values of $1-5$.

Using this technique, Caldwell (26), Caldwell \& Smith (27), Menzer et al (28), and Lloyd et al (29) obtained a linear relationship between time on an isometric task and subjective estimates of both pain intensity and perceived effort expended. With the use of the same technique, subjective estimates of effort were also found to be linearly related to endurance time during treadmill performance (30).

It has been suggested (31) that the self-paced rating procedure used in the aforementioned studies might have contained a timing artefact, in that the subjects might have been judging elapsed time rather than effort expended. The relationship between subjective estimates and time on the task would attain spurious linearity if the subjects of these studies were matching intervals on the time dimension.

The suggestion of spurious linearity is indirectly supported by the results of a recent experiment by Kilbom et al (31), in which static exercise was performed as a $90^{\circ}$ elbow flexion at $25 \%$ of maximal voluntary contraction force, either until exhaustion or for a duration of $20-80 \%$ the of maximal endurance time. In this experiment the subjects were requested to rate effort expended after the exercise had been interrupted by the investigator. Furthermore, the subjects rated effort expended only once at the end of each submaximal test, and only one submaximal test was performed each experimental day. This procedure was adopted to minimize the possible effect of time on the ratings. Under these conditions, the relation of perceived effort expended and time on task was not linear. Thus 15 out of 18 subjects overestimated effort expended as related to endurance time, or, in other words, they underestimated their maximal static endurance capacity during the task.

As has already been mentioned, a category scale with numerical values from 1 to 5 has been used in some investigations to collect estimates of the intensity of pain felt during prolonged physical work. In these studies the researchers also made use of the self-paced technique already described. The value of 1 was defined as constituting the first noticeable pain, and the value of 5 as intolerable pain, ie, the pain perceived at the end of the endurance task. In the experiment by Ljungberg et al (8) reviewed previously, subjects were also required to rate pain intensity on a 5-point rating scale ranging from "no pain" to "intolerable pain." In this experiment no subject rated perceived pain using the highest pain intensity of the scale at maximal endurance time, which clearly indicated that perceived pain in the local muscles was not the primary factor limiting endurance in the task. Considering these results, it seems clear that perceived pain intensity, especially if assessed at submaximal endurance time, 
would be a poor predictor of the static endurance capacity of the individual.

The use of the rating scales for expended effort and perceived pain in the study of perceived exertion during physical exercise has produced results somewhat less convincing than those obtained by the use of ratioscaling techniques or the RPE. The relation between effort expended and time on task in prolonged physical work or endurance tasks has not been as well explored and documented as, eg, the relation between perceived exertion and work load.

\section{Acceptable work load}

Three main approaches can be used to determine how much load a worker can handle safely. They are based on estimations of biomechanical, physiological, and perceptual stresses.

The biomechanical models $(32-34)$ emphasize the loads on the musculoskeletal system during the lifting task and provide estimations of the forces and torques generated during the lifting task. The limitations of the biomechanical models are primarily that they do not directly predict work capacity, that they are static in nature, and that they have only considered work on the sagittal plane.

The physiological models $(35,36)$ are based on predictions of the metabolic energy expenditure in lifting and in other manual materials handling tasks. As in the case of the biomechanical models, the physiological models are restricted to lifting in the sagittal plane alone. Moreover, these models make assumptions with regard to the additivity or comparability of energy costs for different tasks which have not been validated.

As an alternative or a complement to the biomechanical and physiological models, lifting capacity prediction models have been developed on the basis of a psychophysical approach. These models are among the few available for determining the maximum acceptable level of work load in manual materials handling tasks.

The method used resembles in some respects one of the basic psychophysical methods, ie, the method of adjustment, used to determine perceptual threshold (37). The procedure is to ask the subject to adjust his or her work load, ie, the weight of the material to be handled or the frequency of the operation, to the maximum he or she can perform without falling into overexertion or excessive fatigue. The subject is told to "imagine you are on piece-work, getting paid for the amount of work you do, but working a normal 8-hour shift that allows you to go home without feeling bushed, unusually tired or weakened.' Following the procedure used in the method of adjustment, the subject will start with a high work load on one occasion and with a low work load on another. The two results are then averaged. Generally the subject is given 20 40 min to adjust the work load.

Undoubtedly the psychophysical approach is very attractive in its simplicity and deserves serious attention. The applicability of the method relies on the assumption that the subject is able to estimate with some accuracy his or her highest work load. Furthermore, it is assumed that the work loads accepted by subjects under conditions which simulate real work are lighter than the loads leading to manual handling injuries among workers. However, as yet, it is not known to what extent these assumptions are valid.

Using this technique, some researchers (38-43) have systematically collected estimates of work load during different manual materials handling activities standardized and simulated in the laboratory. The results of a series of studies using the psychophysical approach have been summarized and integrated by Snook (44). In his report Snook presents a series of tables indicating the maximum weights predicted to be acceptable to 10 , 25,50 , and $90 \%$ of the male and the female working populations. Similar tables have also been published by Ayoub et al (45), who combined their own data with the data published by Snook (44).

According to Snook (44), a proper use of these tables can reduce the occurrence of musculoskeletal injuries more effectively than selecting the workers for the job or training the workers to lift properly. Currently, however, there is no general agreement concerning the validity of these estimates. Thus Mital (46), studying lifting work, was not able to validate the assumption that people can estimate the amount of weight they can actually lift $8 \mathrm{~h}$ a day based on the effort they perceived in $20-30 \mathrm{~min}$ of work. He also noted that the psychophysical method tends to overestimate the maximum acceptable weight of lift.

Recent studies $(8,47)$ have shown that subjects tend to select higher work loads when adjusting the frequency of the operation performed than when adjusting the weight of the object they have to handle. In one of my own studies using this psychophysical method (8), subjects working with horizontal lifting chose about half as heavy a case as subjects working with vertical lifting at a comparable lifting rate. Similar results have also been reported by Garg $\&$ Badger (48). They found that an increase in the asymmetry of a lifting task was always followed by a decrease in the work load preferred by the subject.

To test the reliability and validity of this psychophysical method, my colleagues and I have performed three experiments $(8,10)$. The concept of maximum acceptable work load is presented in the following discussion in light of the results of these experiments.

The first experiment (8) has already been referred to in connection with the illustration given on the use of the method of magnitude estimation. In this experiment, following the described procedure, five brewer's draymen estimated the maximum acceptable work load 
of a horizontal lifting task lasting 36 and $18 \mathrm{~s}$ with two workplaces.

The subjects had no difficulty in following the instructions. Thus it only took about $10 \mathrm{~min}$ for them to make their choice of maximum acceptable weight. The choices were consistent irrespective of whether a subject had started with 5 or $25 \mathrm{~kg}$ in the case. The intersubject rankings with regard to the maximum acceptable weight were identical for all the trials and therefore indicated that the reproducibility of the results was very satisfactory. However, the interindividual variation was considerable and did not appear to be related to the subjects' physical characteristics, eg, body size, muscle strength, and aerobic power. Thus the lowest weight $(5.3 \mathrm{~kg})$ was selected by a relatively powerfully built person with considerable muscle strength and high aerobic power, whereas the heaviest weight $(17.0 \mathrm{~kg})$ was chosen by a much shorter and lighter subject with less muscle strength and lower aerobic power.

Other results of this experiment should also be taken into consideration when the validity of the psychophysical approach is assessed. First, when the lifting rate was doubled, the average weight selected was only lower by $11 \%$, although the subjects performed $74 \%$ more work. This result indicates that physiological factors may not be the primary determinants of the work loads selected. Second, in a study of vertical lifting with the same instructions and comparable lifting rate (49), the subjects preferred to work with about twice as heavy a case as in the present study. This result clearly indicates that horizontal lifting, which is very common in real materials handling situations, imposes a greater strain than vertical lifting. This fact would suggest that the values of maximum acceptable work loads collected in standardized lifting situations only have a very limited representativity.

Two groups of subjects participated in the second experiment (10). One group consisted of eight warehouse workers with occupational experience of lifting work, and the other group consisted of eight office employees without such experience. A diagonal lifting task was used in this experiment. It consisted of four consecutive lifts. The first lift was from a table on the left side (knuckle height) to a shelf on the right side (knee height). The second lift was moving the case back to the table. The third lift was from the table on the left side (knuckle height) to a shelf on the right side (shoulder height). The fourth lift, concluding the lifting cycle, was moving the weight back to the table.

During the first part of the experiment the subjects performed the lifting task repeatedly using five weights and five work paces. On this occasion the aim was to study the relation between the objective and subjective work loads. As expected from the results of the previous experiment, the perceived work load for this type of lifting task was found to be a positively accelerated function of the objective work load, as defined by the weight lifted and by the work pace.
During the second part of the experiment the subjects selected acceptable weights for this lifting exercise at three different work paces and acceptable work paces for three different weights. An analysis of the results obtained revealed that there were large and systematic differences in the selection of work loads between two groups of subjects who had been instructed by two different experimenters. A thorough investigation afterwards revealed that this bias was due to the fact that during the lifting task one of the instructors had reminded the subjects to adjust the work load if they felt like it. No such action was taken by the other experimenter. Thus reminding some of the subjects that they could adjust the work load any time they wanted produced a systematic increase in the maximum acceptable work load finally selected by the subjects. This result clearly indicates that the data collected by the use of the psychophysical approach are highly dependent on the procedure adopted for instructing the subjects. It seems evident that this characteristic of the method must constitute a definite limitation to its applicability.

In the third experiment (10) two new groups of subjects were chosen following the same criteria as in the previous experiment. The lifting task and the general procedure of this experiment was the same as in the previous experiment. However the instructions given the subjects were further standardized and all the subjects were instructed by the same experimenter. The subjects selected acceptable weights at three different work paces and acceptable work paces for three different weights. They also selected an acceptable work load by changing both the weight and the work pace. For each work load selected, the subjects rated perceived exertion according to the RPE scale (13). The entire procedure was then repeated, the replication of each selection of work load being retained.

On the whole, the reproducibility of the selected work loads was satisfactory also in this experiment. However, it was apparent that selecting the work pace was an easier and more reliable task than selecting a weight.

Two features of the results of the present experiment are relevant for the assessment of the validity of published data concerning maximum acceptable work loads collected with the psychophysical approach. One feature is that occupational experience of lifting work had a negative effect on the selection of work loads. Thus the maximum acceptable work loads selected by the warehouse workers were systematically lower than those selected by the office employees. This phenomenon was also clearly evident when subjects were left free to select both the weight and the work pace in the same trial. Under these conditions the warehouse workers selected a $78 \%$ lower weight and $87 \%$ lower work pace on the average. Although they selected lower work loads, the warehouse workers consistently rated overall perceived exertion and the perceived exertion on 
the arms and shoulders, the back and the legs higher than the office employees. The other feature of significance, with regard to the validity of the method, is that the subjects' physical characteristics, eg, body size, muscle strength, and aerobic power, showed no relation to the work loads they selected.

To summarize, the results of the three experiments raise questions concerning the representativity of psychophysical data collected in the laboratory regarding maximum acceptable work loads for manual materials handling operations. Furthermore, as the experiments have shown, it is difficult to reach a satisfactory understanding of the significance and implication of the concept of maximum acceptable work loads for lifting tasks. The results indicate that the process of selecting a work load might be governed both by cognitive and motivational factors and by perceptual stimuli.

\section{Concluding remarks}

Independent of the specific psychophysical method used to measure it, the perception of effort should be interpreted as constituting a "summing up" of the influence on the organism from all structures under stress during physical work. Although there is no objective counterpart to this perceptual phenomenon, the perception of effort during physical work not only has a psychological validity, but it also reflects real conditions such as the interplay between the requirements of the physical task and the capacity of the individual. No single technique, of those described in this paper as available for measuring subjective reactions during physical work performance, can be considered generally superior to the others.

Undoubtedly, a ratio scaling method such as magnitude estimation is particularly suitable for studying the relation between the perception of effort and the objective work load for a specific physical task. However, in practical settings where an assessment of the physical requirements of different work situations is needed or when interest is focused on interindividual or intraindividual comparisons, the use of a rating scale of perceived exertion can have clear advantages over magnitude estimation.

Rating scales of effort expended have been applied to the study of physical work almost exclusively to collect estimates of effort or pain in prolonged exercise or endurance tasks. As yet the reliability and validity of the measurements of effort expended have not been satisfactorily evaluated.

The use of the method of selecting an acceptable work load seems to be restricted to situations in which the objective is to evaluate manual materials handling activities and to generate norms aimed at the prevention of manual handling injuries. Although the validity of the method in this respect has not yet been demonstrated, the method deserves serious attention. There seems to be good reason to investigate the possibility of combining the psychophysical approach with the biomechanical and physiological ones to develop an integrated model capable to predict, and therefore to prevent, musculoskeletal injuries.

\section{References}

1. Borg G. Psychophysical scaling with applications in physical work and the perception of exertion. Scand $J$ Work Environ Health 1990;16(suppl 1):55-8.

2. Stevens JC, Mack JD. Scales of apparent force. J Exp Psychol 1959;58:405-13.

3. Borg G, Dahlström $H$. The perception of muscular work. Umeả (Sweden): University of Umeả, Research Library, 1960. (Publication no 5.)

4. Eisler H. Subjective scale of force for a large muscle group. J Exp Psychol 1962;64:253-7.

5. Gamberale F, Holmér I, Kindblom AS, Nordström A. Magnitude perception of added inspiratory resistance during steady-state exercise. Ergonomics 1978;21: $531-8$.

6. Stevens JC, Cain WS. Effort in isometric muscular contractions related to force level and duration. Percept Psychophys 1970;8:240-4.

7. Cafarelli E, Cain WS, Stevens JC. Effort of dynamic exercise: influence of load, duration and task. Ergonomics 1970;20:147-58.

8. Ljungberg AS, Gamberale F, Kilbom A. Horizontal lifting - physiological and psychological responses. Ergonomics 1982;25:741-57.

9. Ekman G. Two generalized ratio scaling methods. J Psychol 1958;45:287-95.

10. Gamberale F, Ljungberg A, Annwall G, Kilbom $\dot{A}$. An experimental evaluation of psychophysical criteria for repetitive lifting work. AppI Ergon 1987;18:311-21.

11. Borg G. Physical work and effort. New York, NY: Pergamon Press Ltd, 1977. (Wenner-Gren Center international symposium series; vol 28.)

12. Borg G, Ottoson D. The perception of exertion in physical work. London: McMillan Press, 1986. (Wenner-Gren Center international symposium series; vol 46.)

13. Borg G. Physical performance and perceived exertion. Lund (Sweden): Gleerup, 1962. (Studia psychologica et pædagogica, series altera, investigations XI.)

14. Borg G. Subjective effort in relation to physical performance and working capacity. In: Pick HL, ed. Psychology: from research to practice. New York, NY: Plenum, 1978:333-61.

15. Pandolf KB, Cafarelli E, Noble BJ, Metz F. Perceptual responses during prolonged work. Percept Mot Skills 1972;35:975-85.

16. Gamberale F, Holmér I. Heart rate and perceived exertion in simulated work with high heat stress. In: Borg G, ed. Physical work and effort. New York, NY: Pergamon Press, 1977: 323-32. (Wenner-Gren Center international symposium series; vol 28.$)$

17. Henriksson J, Knuttgen HG, Bonde-Pedersen F. Perceived exertion during exercise with concentric and eccentric muscle contractions. Ergonomics 1972;15:53742.

18. Pandolf KB, Noble BJ. The effect of pedalling speed and resistance changes on perceived exertion for equivalent power outputs on the bicycle ergometer. Med Sci Sports $1973 ; 5: 132-6$.

19. Ekblom B, Goldbarg AN. The influence of training and other factors on the subjective rating of perceived exertion. Acta Physiol Scand 1971;83:399-406.

20. Sjöberg $\mathrm{H}$, Frankenhaeuser $\mathrm{M}$. Interactions between heart rate, psychomotor performance and perceived effort during physical work as influenced by beta adrener- 
gic blockade. Biol Psychol 1979;8:31-43.

21. Morgan WP. Psychological factors influencing perceived exertion. Med Sci Sports 1973;5:97-100.

22. Gamberale F, Strindberg L, Wahlberg I. Female work capacity during the menstrual cycle: physiological and psychological reactions. Scand J Work Environ Health $1975 ; 1: 120-7$.

23. Bar-Or O, Skinner JS, Buskirk ER, Borg G. Physiological and perceptual indicators of physical stress in 41 to 60 year-old men who vary in conditioning level and in body fatness. Med Sci Sports 1972;96:96-111.

24. Noble BJ, Metz KF, Pandolf KB, Cafarelli E. Perceptual responses to exercise: a multiple regression study. Med Sci Sports 1973;5:104-9.

25. Gamberale F. Perceived exertion, heart rate, oxygen uptake and blood lactate in different work operations. Ergonomics 1972;15:545-54.

26. Caldwell LS. The scaling of effort produced by strenuous isometric muscle contractions. US Military Research Laboratory, 1967. (USAMRL report no 749.)

27. Caldwell LS, Smith RP. Subjective estimation of effort, reserve and ischemic pain. US Military Research Laboratory, 1967. (USAMRL report no 730.)

28. Menzer J, Smith P, Caldwell LS. Selfpaced and irregular methods of subjective estimation of pain. Psychonomic Sci 1969;15:287-92.

29. Lloyd AJ, Voor JH, Thieman TJ. Subjective and electromyographic assessment of isometric muscle contractions. Ergonomics 1970;13:685-69.

30. Lloyd AJ, McClaskey EB. Subjective assessment of effort in dynamic work. J Mot Behav 1971;3:49-54.

31. Kilbom $\AA$, Gamberale F, Persson J, Annwall G. Physiological and psychological indices of fatigue during static contractions. Eur J Physiol 1983;50:179—83.

32. Chaffin DB. A computerized biomechanical model: development of and use in studying gross body actions. J Biomech 1969;2:429-41.

33. El Bassoussi MM. A biomechanical dynamic model for lifting in the sagittal plane [Doctoral dissertation]. Lubbock, TX: Texas Tech University, 1974.

34. Fisher B. A biomechanical model for the analysis of dynamic activities [Master's thesis]. Ann Arbor, MI: University of Michigan, 1967.

35. Ảberg V, Elgstrand $\mathrm{K}$, Margnus $\mathrm{P}$, Lindholm A. Analysis of components and prediction of energy expenditure in manual task. Int J Prod Res 1968;3:189-96.
36. Garg A. A metabolic prediction model for manual material handling jobs [Doctoral dissertation]. Ann Arbor, MI: University of Michigan, 1976.

37. Engen T. Psychophysics: discrimination and detection. In: Kling JW, Riggs A, ed. Woodworth Schlosberg's experimental psychology. London: Methuen, 1971.

38. Ayoub MM, Dryden R, McDaniel J, Knipfer MR, Dixon D. Predicting lifting capacity. Am Ind Hyg Assoc J 1979;40:1079-84.

39. Ciriello VM, Snook SH. The effects of size, distance, height and frequency on manual handling performance. In: Boise EJ, Miller JM, ed. Proceedings of the Human Factors Society 22nd annual meeting. Detroit, $\mathrm{MI}$ : Human Factors Society, 1978.

40. Garg A, Saxena U. Effects of lifting frequency and technique on physical fatigue with special reference to psychophysical methodology and metabolic rate. Am Ind Hyg Assoc J 1979;30:894-903.

41. Leggs SJ, Myles WS. Maximum acceptable repetitive lifting workloads for an 8-hour work-day using psychophysical and subjective rating methods. Ergonomics $1981 ; 24: 907-16$.

42. Snook SH, Ciriello VM. Maximum weights and workloads acceptable to female workers. J Occup Med 1974;16:527-34.

43. Snook SH, Irvine $\mathrm{CH}$. Maximum frequency of lift acceptable to male industrial workers. Am Ind Hyg Assoc J 1968;29:531-6.

44. Snook SH. The design of manual handling tasks: the ergonomics society lecture. Ergonomics 1978;21:96385 .

45. Ayoub MM, Mital A, Shibab S, Asfour SS, Bethea NI. Review, evaluation and comparison of models for predicting lifting capacity. Hum Factors 1980;22:257-69.

46. Mital A. Maximum frequencies acceptable to males for one-handed horizontal lifting in the sagittal plane. Hum Factors 1983;25:563-71.

47. Nicholson LM, Legg SJ. A psychophysical study of the effects of load and frequency upon selection of workload in repetitive lifting. Ergonomics 1986;29:903-11.

48. Garg A, Badger D. Maximum acceptable weights and maximum voluntary isometric strengths for asymmetric lifting. Ergonomics 1986;29:879-92.

49. Snook SH, Irvine $\mathrm{CH}$, Bass SF. Maximum weights and workloads acceptable to male industrial workers. Am Ind Hyg Assoc J 1970;32:579-86. 